\title{
Use of computed tomography-single-photon emission computed tomography fusion for diagnosing painful facet arthropathy
}

\author{
Technical note
}

\author{
Matthew McDonald, M.D., ${ }^{1}$ Robert CoOper, M.D., ${ }^{2}$ AND Michael Y. Wang, M.D. ${ }^{3}$ \\ Departments of ${ }^{1}$ Neurological Surgery and ${ }^{2}$ Radiology, Royal Adelaide Hospital, Adelaide, Australia; \\ and ${ }^{3}$ Department of Neurological Surgery, Keck School of Medicine, University of Southern \\ California, Los Angeles, California
}

\begin{abstract}
$\checkmark$ Facet disease is believed to play a major role in axial low-back pain and may prove in the future to be an important indication for posterior dynamic stabilization. However, the lack of good diagnostic tests and imaging methods for identifying this condition have made this entity obscure. Although single-photon emission computed tomography (SPECT) imaging is a highly sensitive and specific test, the images frequently lack adequate resolution, whereas computed tomography $(\mathrm{CT})$ provides excellent resolution but lacks specificity.

Thirty-seven patients with back pain clinically attributable to facet disease underwent CT-SPECT fusion imaging of the lumbar spine. The SPECT images were obtained using a dual-head gamma camera equipped with VXGP highresolution collimators using a $20 \%$ energy window centered at $140 \mathrm{keV}$ and a $360^{\circ}$ rotation totaling 128 projections at 16 seconds each. Transaxial CT images were transferred in the Digital Imaging and Communications in Medicine format to provide proper image overlay in the axial, sagittal, and coronal planes. Scanning for both modalities was performed using standard patient positioning. Patients with concordant images and symptoms then underwent joint injection and/or rhizotomy, which was performed by an independent physician.

Image fusion was successfully performed in all patients, and the image quality allowed definitive localization of the "hot" lesion in all cases, in contrast to conventional high-resolution SPECT scanning, which often led to problems differentiating L4/5 and L5/S1. In patients with solitary lesions, injection led to definitive pain resolution, even if temporary, in all cases with anesthetic blockade.

The CT-SPECT scanning modality combines the virtues of functional and anatomical imaging, aiding the clinician in making the diagnosis of painful facet arthropathy. This modality may prove useful for the selection of patients who are candidates for posterior dynamic stabilization.
\end{abstract}

KEY WORDS $\bullet$ low-back pain $\bullet$ facet $\bullet$ lumbar spine $\bullet$
single-photon emission computed tomography $\bullet$ computed tomography

$\mathrm{P}$ OSTERIOR dynamic stabilization systems are being developed for the treatment of numerous disorders of the lumbar spine. One area in which these devices may be applicable is the surgical treatment of facet joint disease. Although the current mainstay of treatment for facet-related pain remains intraarticular injections and dorsal branch rhizotomies, both techniques suffer from significant drawbacks. Injections often provide only shortterm relief, necessitating numerous, repeated treatments in the patient's lifetime. Rhizotomy of the dorsal ramus, which innervates the facet joint capsule, carries only a moderate efficacy rate, and also frequently requires repeated treatments. Fusion is another alternative, but this

Abbreviations used in this paper: $\mathrm{CT}=$ computed tomography; SPECT $=$ single-photon emission CT; VAS = visual analog scale. has not been well studied and probably represents an overly aggressive approach, particularly if the intervertebral disc is in good condition.

\section{Facet Arthropathy and Posterior Dynamic Stabilization}

Posterior dynamic stabilization devices appear promising for the treatment of facetogenic pain for two reasons. First, many of the devices are designed to "unload" the facet joints, particularly at the extremes of motion (primarily rotation, lateral bending, and extension) where the facet joints are subject to the most biomechanical stress. This unloading may be sufficient to allow the repair and restorative mechanisms of the body to reduce the degree of inflammation, without the permanent sequelae of inter- 
segmental fusion. Second, total facet replacement devices allow for complete removal of the facet that is the pain generator but allow for the preservation of the remaining functional segmental unit of the spine.

\section{Diagnosing Facet Arthropathy}

Identifying the cause of axial low-back pain in a patient can be a difficult problem for the clinician. Many potential offending structures can be implicated, including the intervertebral disc, back muscles, nerve roots, and facet joints. Although a great deal of emphasis has been placed on the intervertebral disc as a pain generator, there is a growing body of evidence to suggest that the posterior articular structures play a major role in select patients. ${ }^{1}$ This facetogenic pain syndrome was first described by Ghormley ${ }^{8}$ in 1933, but controversy regarding this disorder exists because of disagreement over its pathophysiological features, diagnosis, and treatment., ${ }^{2,6}$ In particular, the lack of good diagnostic methods makes this entity obscure.

As with most diseases, facet disease can be suggested based on the patient's history and physical examination. Pain that worsens with extension maneuvers and focal tenderness over a particular joint can serve as a diagnostic clue. Imaging modalities such as CT scanning and magnetic resonance imaging give the clinician an anatomical assessment of the structure of the facet joint with fine detail. ${ }^{3,9,14}$ However, the high prevalence of facets that appear to be abnormal and diseased in asymptomatic individuals reduces the specificity of these anatomical imaging studies.

\section{Rationale for Fusion Imaging}

Nuclear medicine studies provide a nonstructural alternative method for assessing spinal pathological entities; for example, SPECT scans obtained using intravenous technetium-55 will highlight areas of abnormal bone metabolism. This modality, popularized for the whole-body screening of infectious and neoplastic diseases, can also be used to identify synovial joint arthropathy. ${ }^{10}$ The power of SPECT scanning lies in its sensitivity and specificity for functional evaluation of the facet joints. However, even high-resolution studies lack the imaging sharpness that may be necessary to identify the level of disease clearly. The small size and proximity of the articular joints, coupled with variations in the segmentation pattern of the lumbar spine, make accurate identification of the "hot facet" absolutely critical if a targeted intervention is planned.

Previous reports have described the use of fusion imaging to capitalize on the particular strengths of different neuroimaging modalities. The first reports on fusion imaging were described in 1989 and involved the combination of positron emission tomography, CT scanning, and magnetic resonance imaging for evaluating intracranial neoplasms. ${ }^{15}$ There is now significant interest in fusion imaging, particularly in the field of oncology. ${ }^{11,12,16-18}$ These hybrid images have now been used to enhance the accuracy of cancer detection and improve the targeting of invasive surgical procedures, and the precision of fusion imaging using commercially available software has been ascertained with fiducial registration studies to be at the millimeter level. ${ }^{13}$

Both hardware- and software-based techniques have been developed for fusion imaging. Hardware-based techniques use dual scanning so that the two types of images are acquired simultaneously. This approach is appealing because it obviates some of the problems associated with image coregistration. However, the currently available dual scanners provide low-quality CT images, and software-based systems, which require matching of separately acquired images, are currently more popular.

The application of fusion imaging to degenerative spine disease is in its infancy. In this report we describe our experience using CT-SPECT fusion imaging to identify painful arthropathic facet joints, a technique we have found quite helpful for use in targeting injection therapies.

\section{Fusion Imaging Method}

Fusion imaging of the lumbar spine was obtained using standard techniques for SPECT and CT scanning, but care was taken to place the patient in a standardized supine position during acquisition of the image data. The SPECT scans were obtained using a dual-head gamma camera (ADAC Forte) equipped with VXGP high-resolution collimators. Acquisitions were performed using a 20\% energy window centered at $140 \mathrm{keV}$. The data acquisitions included a $360^{\circ}$ noncircular rotation totaling 128 projections and a matrix of $128 \times 128$. Acquisition time for each projection was 16 seconds. The CT scans were obtained as a volume set from T-12 to S-2 (Toshiba Acquilon 16) at $135 \mathrm{kV}$ and $250 \mathrm{~mA}$, and a 1-second rotation time was used. A field of view of 150 and small focus were used. One-millimeter slices were then reconstructed $0.8 \mathrm{~mm}$ apart by using a soft-tissue algorithm.

Prior to image fusion, the CT scans were transferred in a suitable Digital Imaging and Communications in Medicine format to a Vitrea processing workstation (ADAC Pegasus). Transaxial slices were converted to volume-rendered images on a 256 matrix size. The data were transferred using ADAC proprietary software under the supervision of a radiologist. Only minimal positional differences in the lumbar spine were noted between the CT and SPECT scans due to the standardized positioning of the patients during image acquisition. The SPECT scans were then adjusted so that the filter chosen would typically have a higher cutoff than used for standard nuclear imaging. This prevents the size of any "hot spot" from exaggerating the image. The CT and SPECT scans were then superimposed, using views in all three planes to ensure proper positioning and sizing. Whereas it is not possible to perform this superimposition accurately based on the details of the spinal anatomy, the use of clearly identifiable homologous structures makes this feasible. For example, use of the sacroiliac joints, pelvis, or origin of the ribs allows definitive image superimposition. The SPECT scans were then finalized in RGB color format.

\section{Case Series}

Over a period of 12 months, 37 patients underwent 41 procedures, with a total of 117 lumbar facets injected. Prior to the intervention, all patients underwent CTSPECT fusion imaging. In all cases the scans were obtained without difficulty and a match was made between the two modalities without ambiguity. All patients under- 


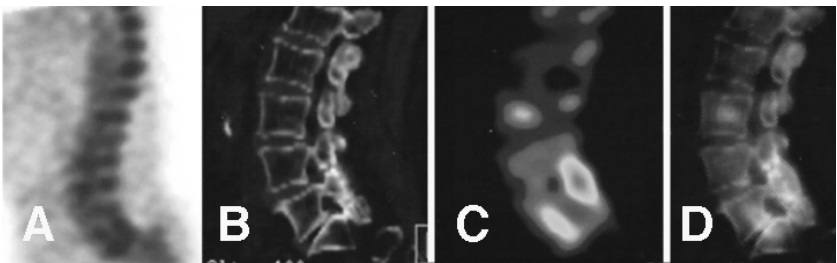

FIG. 1. Neuroimages obtained in a 76-year-old woman who presented with severe back pain. A: Lumbar bone scan demonstrating increased ${ }^{99} \mathrm{Tc}$ radiotracer uptake in the lower lumbar spine. B: Conventional CT scan revealing diffuse spondylosis with disc space narrowing at L5/S1. C: The RGB color SPECT image that will be combined with $\mathrm{CT}$ data to provide a fusion image. D: The resulting CT-SPECT image demonstrates clearly that the ${ }^{99} \mathrm{Tc}$ radiotracer has localized to the L5/S1 disc space and the L4/5 facet joints. Bilateral L4/5 intraarticular facet joint injections with a local anesthetic and steroids resulted in improvement of symptoms for more than 3 months.

went injections targeted to specific levels and sides based on nuclear medicine imaging (Fig. 1). Targeting was accomplished using intraprocedural fluoroscopic imaging. Twenty-five of the patients were women and 12 were men. The mean age was 68 years, with a range of 40 to 90 years. A mean of 2.85 joints was injected per procedure.

The mean duration of symptoms prior to treatment was 38 months. The mean preprocedural VAS score was $7.2 \pm$ 1.3 on a scale of 1 to 10 . The mean postprocedural VAS score was $2.8 \pm 1.6$. This reflected a mean improvement of $4.4 \pm 1.6$ VAS points. Only one patient experienced no improvement following the injection. The mean duration of pain relief was $2.2 \pm 2.2$ months. There were no procedure-related complications. (The preceding values are given as the mean \pm standard deviation.)

\section{Discussion}

The SPECT scanning modality is gaining popularity for diagnosing facetogenic pain. This modality appears to render highly sensitive identification of any facet arthropathy. In the study by Holder et al., ${ }^{10}$ high-resolution SPECT bone imaging proved to be $100 \%$ sensitive and $71 \%$ specific for a clinical diagnosis of facet disease. In the study by Dolan et al., ${ }^{5}$ positive findings on SPECT scans resulted in a $95 \%$ response rate to targeted injection therapy. However, the poor resolution on a SPECT study can make clear localization of the level difficult.

The CT-based studies can provide excellent anatomical detail, as well as localization of the diseased structure, and classification systems have been developed to quantify the degree of osteoarthritic change in the facet joints. ${ }^{7,19}$ On CT scanning the arthropathy is identified by hypertrophy of the articular processes, cupping of the joint edges, narrowing of the synovial space, and osteophytic overgrowth. However, substantial controversy has arisen over the correlation between abnormal facet morphological features and pain. ${ }^{4}$ In fact, grossly abnormal facets are frequently identified incidentally in patients without back pain.

In this report we describe the fusion of highly sensitive and specific SPECT imaging with anatomically superior CT scans. The application of this technique uses standard imaging hardware found in most hospitals but requires software for image reformatting. These images have allowed us to direct localized injections with a high degree of reliability, obviating the need for injection of neighboring facets to ensure proper coverage of the "hot area." This practice of potentially injecting asymptomatic adjacent joints is followed at many centers and probably results in a higher likelihood of pain relief, but it also causes unnecessary trauma and can possibly accelerate degeneration of previously normal joints. We have found CT-SPECT fusion to be highly predictive of a positive pain relief response after targeted anesthetic infiltration of the facet. The technique has been particularly useful in patients with abnormal lumbar segmentation patterns in which lumbarization or sacralization of the vertebrae have made precise localization on SPECT scans more problematic. The application of this technology may result in more reliable diagnosis and treatment of painful facet arthropathy. Proper diagnostic tests and spinal level localization will become increasingly critical as surgical interventions, such as posterior dynamic stabilization, become available.

\section{Disclaimer}

There were no outside funding sources for this manuscript.

\section{References}

1. Badgley C: The articular facets in relation to low-back pain and sciatic radiation. J Bone Joint Surg 23:481-496, 1941

2. Carette S, Marcoux S, Truchon R, Grondin C, Gagnon J, Allard Y, et al: A controlled trial of corticosteroid injections into facet joints for chronic low back pain. N Engl J Med 325: 1002-1007, 1991

3. Carrera G, Haughton VM, Syvertsen A, Williams AL: Computed tomography of the lumbar facet joints. Radiology 134: $145-148,1980$

4. Demaerel P, Wilms G, Goffin J, Baert AL: Osteoarthritis of the facet joints and its role in low-back pain: evaluation with conventional tomography. J Belge Radiol 75:81-86, 1992

5. Dolan AL, Ryan PJ, Arden NK, Stratton R, Wedley JR, Hamann W, et al: The value of SPECT scans in identifying back pain likely to benefit from facet joint injection. Br J Rheumatol 35:1269-1273, 1996

6. Dory M: Arthrography of the lumbar facet joints. Radiology 140:23-27, 1981

7. Fujiwara A, Tamai K, Yamato M, An HS, Yoshida H, Saotome $\mathrm{K}$, et al: The relationship between facet joint osteoarthritis and disc degeneration of the lumbar spine: an MRI study. Eur Spine J 8:396-401, 1999

8. Ghormley R: Low back pain with special reference to the articular facets with presentation of an operative procedure. JAMA 101:1773-1777, 1933

9. Grenier N, Kressel HY, Schiebler ML, Grossman RI, Dalinka MK: Normal and degenerative posterior spinal structures: MR imaging. Radiology 165:517-525, 1987

10. Holder LE, Machin JL, Asdourian PL, Links JM, Sexton CC: Planar and high-resolution SPECT bone imaging in the diagnosis of facet syndrome. J Nucl Med 36:37-44, 1995

11. Keidar Z, Israel O, Krausz Y: SPECT/CT in tumor imaging. Technical aspects and clinical applications. Semin Nucl Med 33:205-218, 2003

12. Koral KF, Lin S, Fessler JA, Kaminski MS, Wahl RL: Preliminary results from intensity-based CT-SPECT fusion in I131 anti-B1 monoclonal-antibody therapy of lymphoma. Cancer 80:2538-2544, 1997

13. Mongioli V, Brusa A, Loi G, Pignoli E, Gramaglia A, Scorsetti $\mathrm{M}$, et al: Accuracy evaluation of fusion of CT, MR, and SPECT 


\section{McDonald, R. Cooper, and M. Y. Wang}

images using commercially available software packages. Int J Radiation Oncology Bio Phys 43:227-234, 1999

14. Pathria M, Sartoris D, Resnick D: Osteoarthritis of the facet joints: accuracy of oblique radiographic assessment. Radiology 164:227-230, 1987

15. Pelizzari CA, Chen GT, Spelbring DR, Weichselbaum RR, Chen CT: Accurate three-dimensional registration of CT, PET, and/ot MR images of the brain. J Comput Assist Tomogr 13: 20-26, 1989

16. Profanter C, Wetscher GJ, Gabriel M, Sauper T, Rieger M, Kovacs $\mathrm{P}$, et al: CT-MIBI image fusion: a new preoperative localization technique for primary, recurrent, and persistent hyperparathyroidism. Surgery 135:157-162, 2004

17. Schettino C, Kramer E, Noz M, Taneja S, Padmanabhan P, Lepor H: Impact of fusion of indium-111 capromab pendetide volume data sets with those from MRI or CT in patients with recurrent prostate cancer. AJR Am J Roentgenol 183: 519-524, 2004
18. Schillaci O, Danelli R, Manni C, Capoccetti F, Simonetti G: Technetium-99m-labeled red blood cell imaging in the diagnosis of hepatic hemangioblastomas: the role of SPECT/CT with a hybrid camera. Eur J Nucl Med Mol Imaging 31: 1011-1015, 2004

19. Weishaupt D, Zanetti M, Boos N, Hodler J: MR imaging and $\mathrm{CT}$ in osteoarthritis of the lumbar facet joints. Skeletal Radiol 28:215-219, 1999

Manuscript submitted October 18, 2006.

Accepted November 20, 2006.

Address reprint requests to: Michael Y. Wang, M.D., Department of Neurological Surgery, Keck School of Medicine, University of Southern California, 1200 North State Street, Suite \#5046, Los Angeles, California 90033. myw@usc.edu. 